\title{
Pancreatic Cancer and its Treatment
}

\author{
Manu Mitra*
}

\section{Department of Alumnus with Electrical Engineering, University of Bridgeport, USA}

\section{Introduction}

Pancreas is an organ in the human body that is located in the abdomen. It plays very vital and crucial role in transforming the food we eat into fuel for the human body's cells. It has two main functions; exocrine function that supports in digestion and other is an endocrine function that controls blood sugar.

Cancer begins when cells in the body start to grow out of control. As a matter of fact, cells in nearly any part of the body can become cancer and can spread to other parts of the body. It is very vital to know that if the cancer is an exocrine or endocrine because they have distinct risk, causes and they also have different signs and symptoms; they are diagnosed, treated different ways and have different viewpoint. The two major types of cancer in pancreas are exocrine pancreatic cancer and endocrine pancreatic tumors which are also called as neuroendocrine tumors (Figure 1) [1,2].

Below list discusses various treatment of pancreatic cancer including treatment through nanomedicine from plenty of sources.

\section{Stroma Provides a Novel Method for Treatment of Pancreatic Cancer}

One of the major reason why pancreatic tumors are so resistant to treatment because the "wound" like tissue that surrounds the tumors, that is termed as stroma. It is much denser than other stromal tissues surrounding other tissues. Stromal tissue is understood to contain influences that aid tumor's growth and its survival and most important, in pancreatic cancer, its density is thought to be a factor in avoiding cancer killing drugs from reaching the tumor.

Scientists at Cold Spring Harbor Laboratory made an important step nearer in understanding why pancreatic cancers are so hard to treat. In their investigations reported, organoid technology is taken a new level in which organoids derivative from tumors are for the first time "co-cultured" with one component of stroma tissue in which tumors develop. The outcome is more realistic of what take place in the pancreas cancer, in this case from stroma, can be clearly analyzed.

"We can imagine a pancreas tumor as a big oatmeal cookie with the raisins representing the cancer cells and oatmeal part representing the dense stroma that creates over $90 \%$ of the tumor" was said by David Tuveson, M.D., Ph.D., Director of the Cancer Center at Cold Springs Harbor Laboratory (Figure 2) $[3,4]$.

\section{A New Potential Therapeutic Strategy for Pancreatic Cancer}

Most of the studies have concentrated mainly on tumor cells. Whereas the cells that form the stroma are significantly unknown, in spite of various research groups demonstrating that some components of stroma promote tumor progression. Various other researcher groups have tried to eliminate the most of plentiful cells in the stroma; they are so called cancer associated fibroblasts (CAFs), but they achieved the opposite effect to what it was expected. Tumor continued to advancement with even greater aggressiveness. The explanation of this investigation report was that some of the cells eliminated could have anti-tumorigenic functions.

Therefore, researchers concentrated on a sub population of fibroblasts which are known to play a vital part in inflammation the reason because inflammation nurtures tumor growth. Their task was to analyze all the genes that are otherwise expressed in the stromal fibroblasts of the tumor. The analysis revealed that Saa3 gene was accountable for CAFs assisting tumor cells to advance. And when the scientists eliminated the expression of this particular gene in the CAFs, these cells performed like normal fibroblasts, because they lost the capability to assist tumor cells for further advancement. Researcher managed to reprogram these cells which had been stripped of their pro tumor properties.

"This method may represent a future therapeutic strategy to combine with other strategies such as immunotherapy, chemotherapy or inhibitors against specific indicating routes of the tumor cells and opens the doors to new research at reprogramming the stroma instead of eradicating it" was told by Djurec $[5,6]$.

*Corresponding author: Manu Mitra, Department of Alumnus with Electrical Engineering, University of Bridgeport, 126 Park Avenue, Bridgeport, CT - 06604, USA

Accepted: November 20, 2018

Published online: November 22, 2018

Citation: Mitra M (2018) Pancreatic Cancer and its Treatment. J Gastrointest Hepat Surg 1(1):9-13 


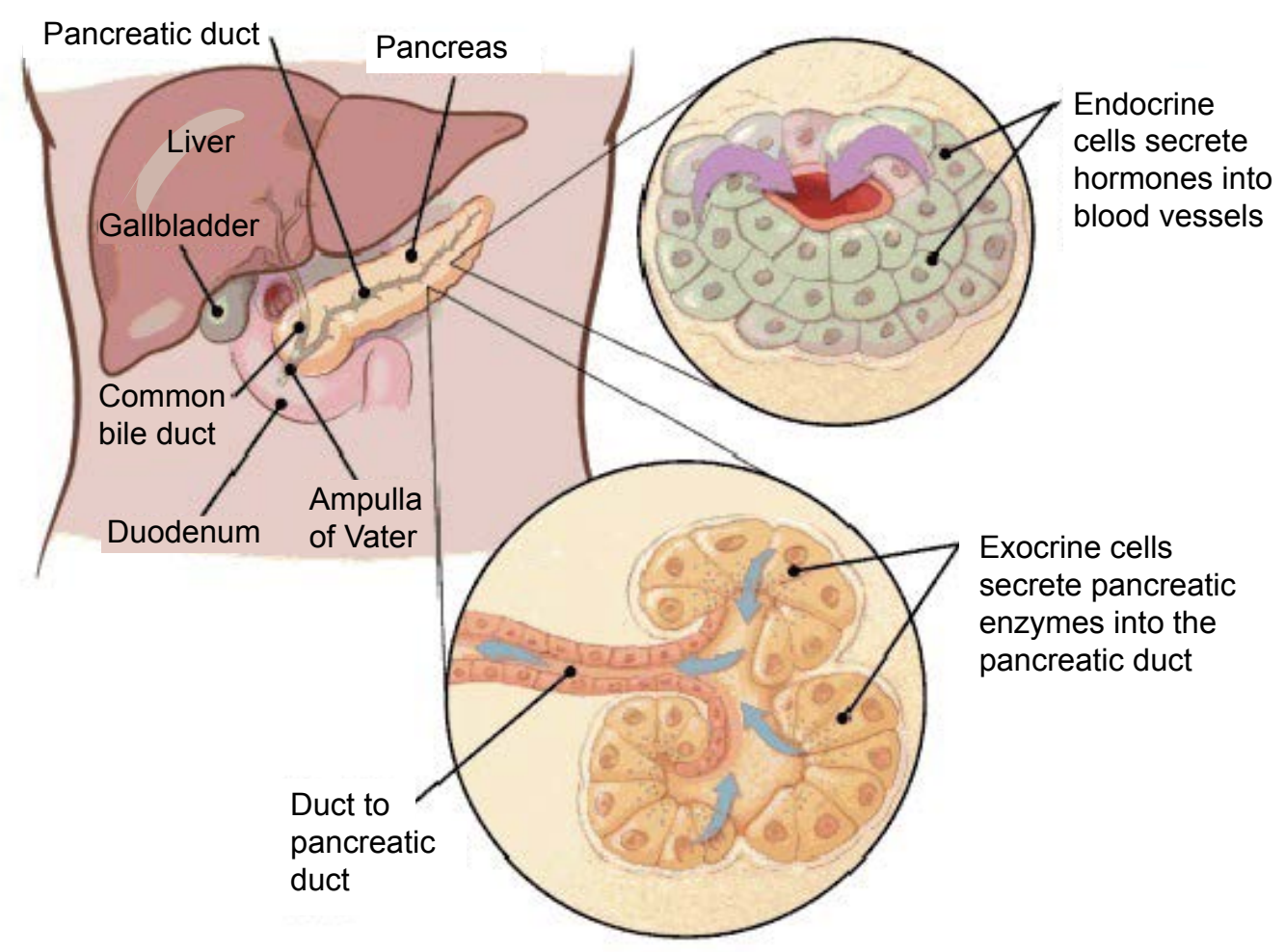

Figure 1: Illustrates the location of endocrine and exocrine pancreas in the human body [2].
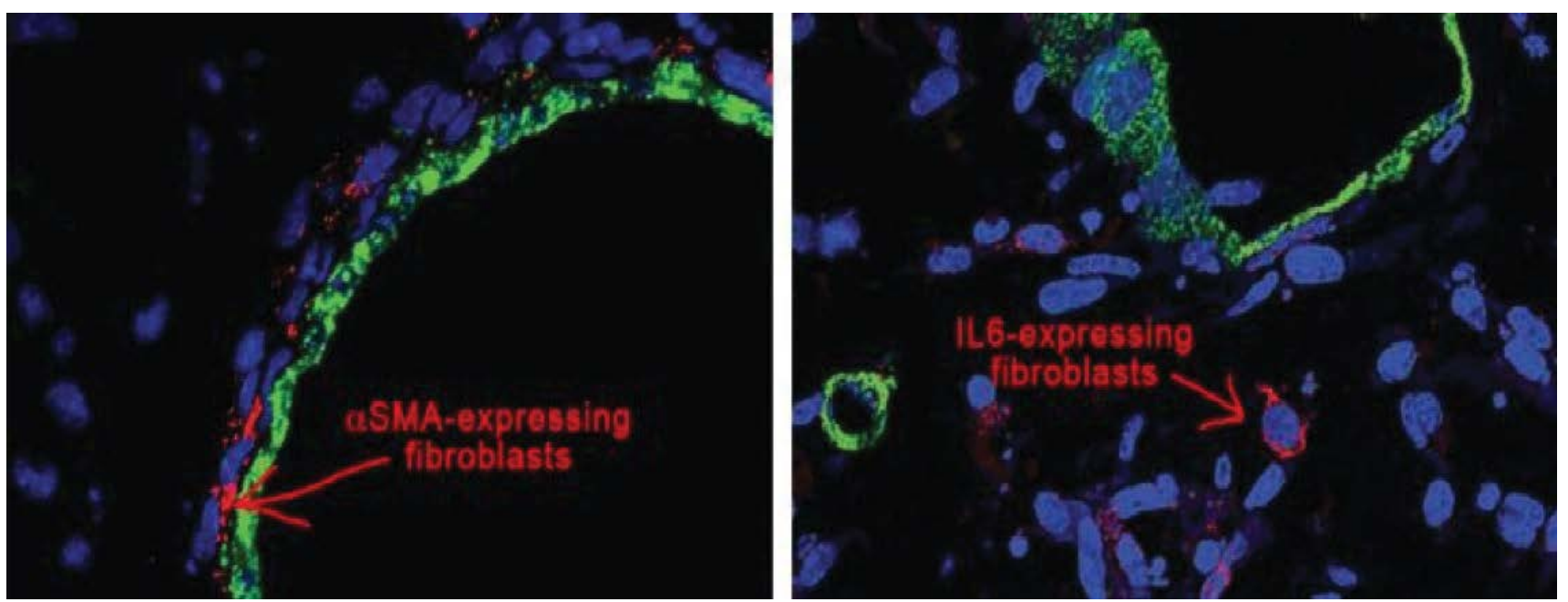

Figure 2: Illustrates dense stromal tissue that envelops pancreatic tumors. One of these difference is seen in left image: fibroblasts (red) close to proliferating cancer cells (green) that express high levels of a protein called aSMA. In the image at right, red color indicates the presence of the immune-signaling molecule IL6, which a second subclass of tumor-related fibroblasts secretes. Image Credit: Tuveson Lab, CSHL [4].

\section{Novel Approach of Treating Pancreatic Cancer}

Scientists at Binghamton University heated and froze tumor cells and observed at the effect using various techniques to decide the level of cell death, on regrowth as well as which cell stress pathways were triggered. According to Van Buskirk, modulating these stress pathways is the significant trail to make heating and freezing ablation process more effective. This could lead to the new development to remove cancerous pancreatic tumors. In addition to cell molecular research, several other group members of the study team are working on improving new catheter technologies to provide this ablative therapy to patients.

"If a very thin catheter can be made to target tumor and if we understand how pancreatic cancer reacts to ablation at molecular level, then we can develop a new therapy that can kill tumor in a very difficult place such as pancreas" was confirmed by Van Buskirk (Figure 3) [7,8].

\section{Nanomedicine for Pancreatic Cancer}

Pancreatic cancer is among most aggressive cancers 

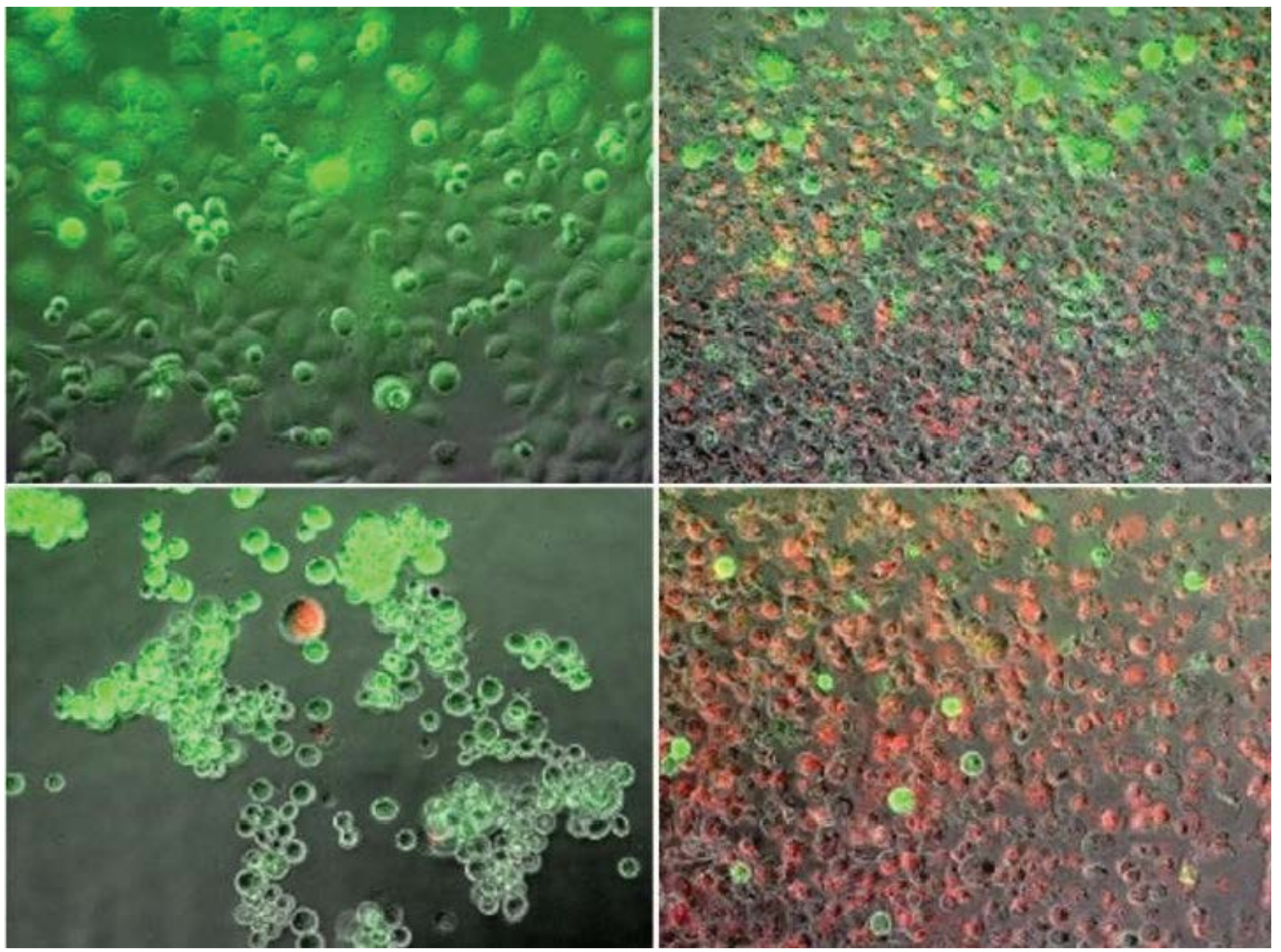

Figure 3: Illustrates pancreatic cancer cells stained with live/dead fluorescence dyes, with live cells in green and dead cells in red. Pictured are control cells (top left), frozen cells (top right), heated cells (bottom left) and DTA treated cells (bottom right). Image Credit: Credit: Kenneth W Baumann [8].

known today. There are significant majority of pancreatic cancer patients die within just a year of diagnosis. Considering the issue research team investigated pancreatic cells and invented an inverse correlation between the signatures of miR-34a, a tumor suppressant and PLK1 a known oncogene. The levels of miR-34a were low in pancreatic cancer mouse models whereas the levels of oncogene were high. This correlation was made clear for such an aggressive cancer. But researcher's team had to understand to see if it was same in humans. Then experts performed RNA profiling and investigations of samples taken from pancreatic cancer patients. The molecular profiling exposed the same genomic pattern that was found earlier in mouse models of pancreatic cancer.

The researchers then devised a new method of nanoparticle that delivers genetic material to a tumor and avoids side effects in surrounding healthy tissues. "The nanoparticle is like a taxi, transporting two important passengers" was confirmed by Prof. Satchi-Fainaro $[9,10]$.

\section{Combating Pancreatic Cancer}

In a study, working with group of expert researchers, Danino proved that bacteria in pancreatic tumors reduce a chemotherapy drug called Gemcitable most commonly used to treat patients who have pancreatic cancer. In preclinical trials and experiments, the team of researchers injected mice with antibiotics to take a note if it would mitigate the effect of bacteria. In more than 70 percent in mice, antibiotics proved effective in killing the bacteria. And if antibiotics are found to kill bacteria in pancreatic patients then chemotherapy may become more effective to patients giving more hope and more life.

"The goal is to use novel techniques for social good and to improve the health of many people" was said by Danino, a biomedical engineering professor at Columbia Engineering $[11,12]$.

\section{Nanomedicine for Treating Pancreatic Cancer}

Scientist performed RNA profiling and analysis of sample taken from pancreatic cancer patients and analyzed the results that an inverse correlation between the signatures of miR-34a, a tumor suppressant and PLK1 a known oncogene. The intensity of oncogene was high. This correlation made sense for an aggressive pancreatic cancer.

Then Scientists devised a new method nanoparticle that 


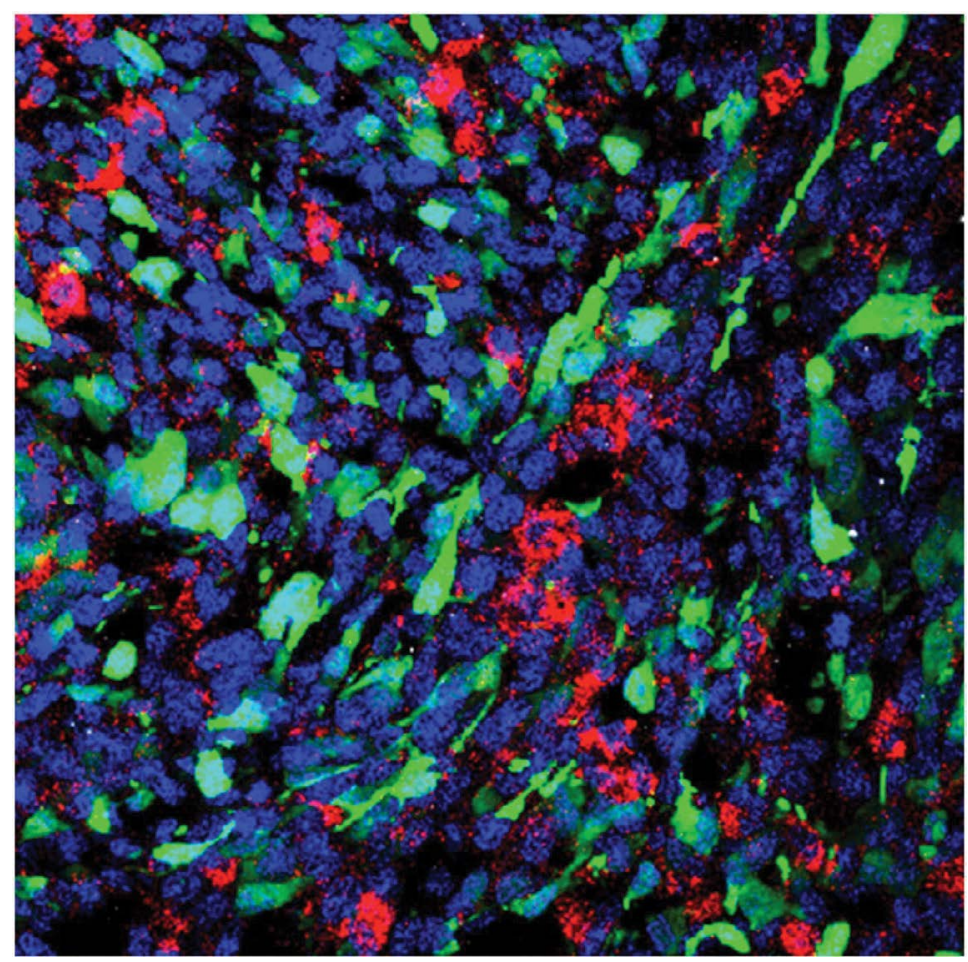

Figure 4: Illustrates experimental nanomedicine approach employs a model of glioblastoma in which nanoparticles (red), injected intracranially, are taken up by tumor cells (green). Image Credit: Eric Song (Yale University, New Haven, CT) [14].
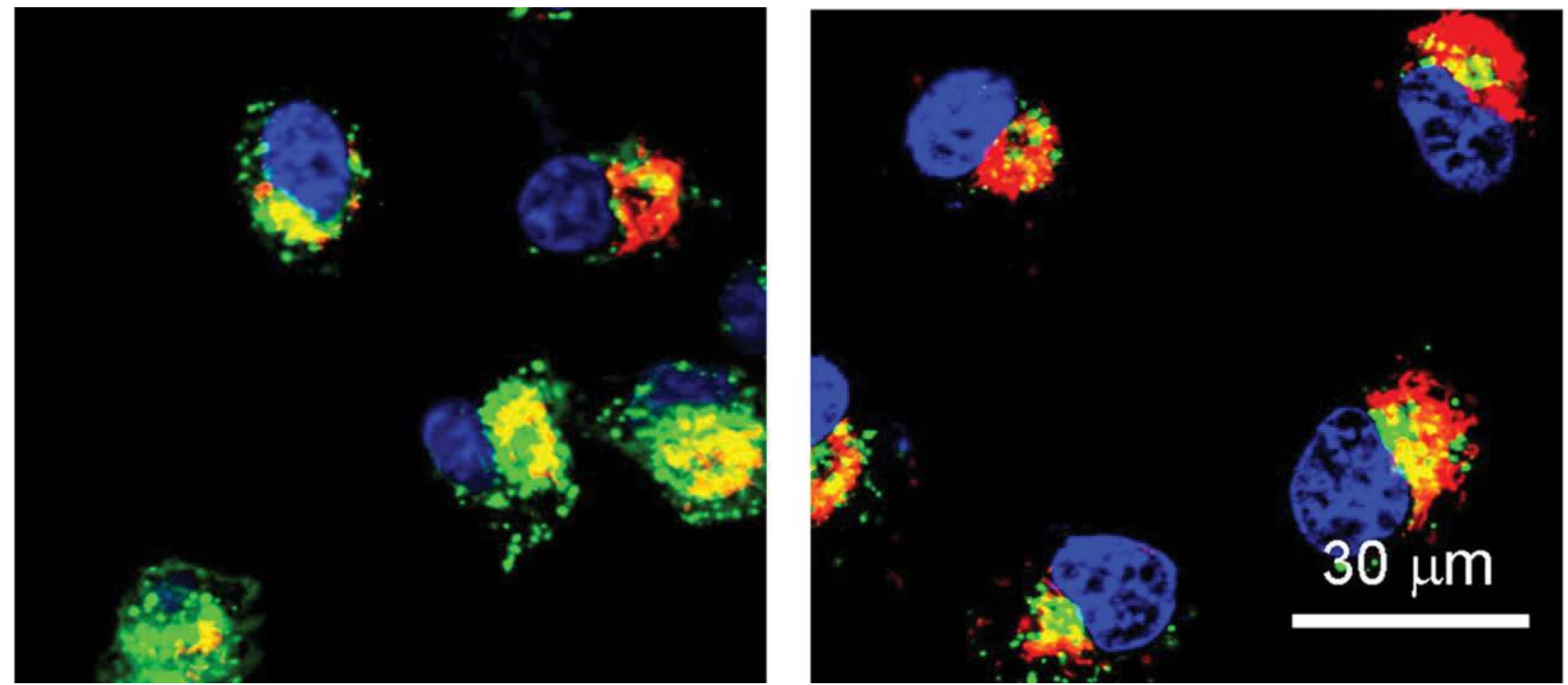

Figure 5: Illustrates confocal laser scanning microscope images show nanoparticles (red) releasing a payload of gene-silencing RNA into cervical cancer cells. Image Credit: Xia-Ding Xu (Harvard Medical School, Boston) [14].

predominantly delivers genetic material to tumor and avoids after effects for neighboring healthy tissues (Figure 4 and Figure 5) $[9,10,13,14]$.

\section{Acknowledgment}

Author would like to thank Prof. Navarun Gupta, Prof. Hassan Bajwa, Prof. Linfeng Zhang and Prof. Hmurcik for their academic support. Author also thanks anonymous reviewers for their comments.

\section{Conflicts of Interest}

There is no conflict of interest as per Author's point of view.

\section{References}

1. http://columbiasurgery.org/pancreas/pancreas-and-itsfunctions

2. https://www.cancer.org/cancer/pancreatic-cancer/about/ what-is-pancreatic-cancer.html 
3. Daniel Öhlund, Abram Handly-Santana, Giulia Biffi, et al. (2017) Distinct populations of inflammatory fibroblasts and myofibroblasts in pancreatic cancer. J Exp Med Jem 214: 579-596.

4. www.sciencedaily.com/releases/2017/02/170223124234.htm

5. Magdolna Djurec, Osvaldo Graña, Albert Lee, et al. (2018) Saa3 is a key mediator of the protumorigenic properties of cancerassociated fibroblasts in pancreatic tumors. Proc Natl Acad Sci USA 115: E1147-E1156.

6. www.sciencedaily.com/releases/2018/02/180201115728.htm

7. Kenneth W Baumann, John M Baust, Kristi K Snyder, et al. (2017) Dual thermal ablation of pancreatic cancer cells as an improved combinatorial treatment strategy. Liver and Pancreatic Sciences 2.

8. www.sciencedaily.com/releases/2018/02/180226131424.htm
9. Hadas Gibori, Shay Eliyahu, Adva Krivitsky, et al. (2018) Amphiphilic nanocarrier-induced modulation of PLK1 and miR-34a leads to improved therapeutic response in pancreatic cancer. Nat Commun 9: 16.

10. www.sciencedaily.com/releases/2018/01/180102114228.htm

11. Leore T Geller, Michal Barzily-Rokni, Tal Danino, et al. (2017) Potential role of intratumor bacteria in mediating tumor resistance to the chemotherapeutic drug gemcitabine. Science 357: 1156-1160.

12. www.sciencedaily.com/releases/2017/10/171005161127.htm

13. Mitra M (2018) Editorial on Advanced Nanomedicine. Int J Nanotechnol Nanomed 2: 1-2.

14. Bourzac K (2016) News Feature: Cancer nanomedicine, reengineered. Proc Natl Acad Sci U S A 113: 12600-12603. 